\title{
MANIFESTAÇÕES CLÍNICAS DA \\ NEUROCISTICERCOSE NA REGIÃO DO \\ SEMI-ÁRIDO DO NORDESTE BRASILEIRO
}

\author{
Maria das Graças Loureiro das Chagas' ${ }^{1}$ Argemiro D' Oliveira Júnior' ${ }^{2}$, José Tavares-Neto ${ }^{3}$
}

\begin{abstract}
RESUMO - Foram analisados 44 prontuários de pacientes com neurocisticercose, procedentes de Campina Grande e de outras cidades do Estado da Paraíba, Brasil, atendidos entre 1990 e 2001. A média de idade ( $\pm D P$ ) foi $20,6 \pm 14,3$ anos, sendo $54,5 \%$ do gênero masculino. Trinta e oito $(86,4 \%)$ pacientes eram procedentes de área urbana. A convulsão foi o sintoma inicial em $90,9 \%$ dos pacientes e a cefaléia em $9,1 \%$. A forma epiléptica ocorreu em $63,6 \%$ dos casos e a forma combinada em $22,7 \%$. A tomografia computadorizada do crânio foi compatível com neurocisticercose em $100 \%$ dos pacientes, revelando calcificações (59,1\%), cistos viáveis $(36,4 \%)$ e cistos em degeneração $(31,8 \%)$, de forma isolada ou em associação. O LCR, realizado em 29 pacientes, mostrou alterações em $25(86,2 \%)$, predominando pleocitose linfomonocitária (100\%), e positividade das reações imunológicas (64,3\%). Em conclusão, a neurocisticercose está presente no Estado da Paraíba, é causa frequente de convulsões em crianças e adultos jovens, e sua ocorrência tanto em área urbana quanto na rural denota o pobre controle do complexo teníase/cisticercose neste Estado.
\end{abstract}

PALAVRAS-CHAVES: neurocisticercose, epidemiologia, epilepsia, tomografia.

\section{Clinical aspects of neurocysticercosis at semi-desert region of Brazilian northeast}

\begin{abstract}
Case sheets of 44 patients with neurocysticercosis, coming from Campina Grande and others cities of Paraíba state, examined between 1990 - 2001 were analyzed. The average age (SD \pm ) was $20.6 \pm$ 14.3 years old, of which $54.5 \%$ were male. Thirty eight patients $(86.2 \%)$ came from urban area. The initial symptom was convulsion in $90.9 \%$ of the cases and headaches in $9.1 \%$. The epileptical form was present in $63.6 \%$ of the cases and the combined form in $22.3 \%$. Computerized tomography of the skull was compatible with neurocysticercosis in $100 \%$ of the cases, showing calcification in (59.1\%), integral cysts in (18.2\%) and in degenerating in (20.4\%), isolated or associated. The LCR performed on 29 patients showed alterations in 25 $(86.2 \%)$ cases, predominating linfomonocitary pleocitose in $100 \%$ of the cases and positive immunological reactions in $64.3 \%$ of the cases. In conclusion neurocysticercosis is a frequent cause for convulsions in children and young adults in this region, and the ocorrence in urbane and rural areas denote the poor control of the taeniases/cistycercosis complex in this state.
\end{abstract}

KEY WORDS: neurocysticercosis, epidemology, epilepsy, tomography.

O homem é o único hospedeiro definitivo da Taenia solium, agente causador da neurocisticercose, doença que está diretamente relacionada às condições sócio-sanitárias da população e, por este motivo muito, prevalente nos países em desenvolvimento (América Latina, África Meridional, Índia e Ásia sub oriental) ${ }^{1,2}$. Nas últimas décadas tem sido encontrada também nos países desenvolvidos, como os Estados Unidos, fato este atribuído às correntes migrató-rias principalmente de pessoas provenientes da América Latina ${ }^{3}$. A sintomatologia da neuro- cisticercose é muito variada e depende de fatores tais como: número, localização, fase evolutiva dos cistos e imunidade do hospedeiro ${ }^{3-6}$, que determinam também a gravidade do quadro clínico apresentado pelo paciente. Os sintomas mais comuns são as convulsões, a cefaléia, os distúrbios psíquicos, a hipertensão intracraniana, entre outros. Podem ocorrer de forma isolada ou associados, determinando diferentes formas de apresentação clínica ${ }^{4,7,8}$.

Devido à riqueza de sintomas que pode causar, o diagnóstico da neurocisticercose depende da reali-

\footnotetext{
${ }^{1}$ Professora ajunta do Departamento de Medicina Interna Social e Preventiva da Universidade Federal da Paraíba - Campus II - Campina Grande - Paraíba PB, Brasil; ${ }^{2}$ Professor adjunto - Doutor do Departamento de Medicina Preventiva da Faculdade de Medicina da Universidade Federal da Bahia, Salvador BA, Brasil (FM/UFBA); ${ }^{3}$ Livre-docente do Departamento de Medicina Interna da FM/UFBA.
}

Recebido 2 Agosto 2002, recebido na forma final 26 Dezembro 2002. Aceito 16 Janeiro 2003

Dra. Maria das Graças Loureiro das Chagas - Rua Edvaldo H.M. Borges 220 - 58103-073 Campina Grande PB - Brasil. E-mail:gracas.loureiro@bol.com.br 
zação de exames de neuroimagem (tomografia computadorizada (TC) e/ou ressonância magnética (RM)), associados ao estudo completo do liquido cefaloraquidiano (LCR), com reações imunológicas específicas para esta doença. Como estes exames são de alto custo, muitas regiões não só do Brasil, mas também dos outros países envolvidos, têm dificuldade de realizá-los, o que com certeza diminui a frequência do diagnóstico e influencia na determinação da prevalência desta doença. Esta situação, comum a várias cidades do Nordeste brasileiro, torna relevante a descrição de casos desta doença ocorridos e confirmados laboratorialmente no Estado da Paraíba, a fim de alertar tanto a comunidade médica como as autoridades sanitárias para o problema. Neste Estado, os únicos dados relativos ao Complexo teníase/ cisticercose publicados até o momento são: os de Gonçalves-Coelho e Coelho ${ }^{9}$, que encontraram a partir de levantamento tomográfico, uma frequência de $1,02 \%$ de TC com lesões sugestivas de neurocisticercose; e os de Pereira et al. ${ }^{10}$, que encontraram uma frequência de $5,7 \%$ de teníase entre crianças de 0 a 8 anos de idade, de uma favela de Campina Grande.

Analisamos 44 casos de neurocisticercose observados em Campina Grande e outras cidade do Estado da Paraíba.

\section{MÉTODO}

Os prontuários de quarenta e quatro pacientes com diagnóstico de neurocisticercose, segundo os critérios de Del Brutto et al.(2001) ${ }^{11}$, atendidos no Ambulatório de Neurologia do Hospital Universitário Alcides Carneiro, de Campina Grande (PB), em consultório particular, no período de 1990 a 2001, foram selecionados e analisados retrospectivamente. Como critérios de inclusão, foram considerados: serem naturais de Campina Grande ou procederem de outras cidades do Estado da Paraíba; apresentarem tomografia computadorizada do crânio com imagem sugestiva de neurocisticercose. Os prontuários selecionados foram revisados e preenchida uma ficha padronizada para coleta dos dados: demográficos (idade, gênero e ocupação); e aqueles referentes à idade de início dos sintomas, hábitos alimentares (ingesta de carne de porco mal cozida e/ou verduras e legumes crus); sintomatologia, tipo de convulsão, exame neurológico, exames complementares realizados (TC, RM, LCR e EEG) e o tratamento instituído. De acordo com estes dados foi estabelecida a forma clínica apresentada, o diagnóstico segundo Sotelo et al. ${ }^{12}$ (1985) e o grau de certeza diagnóstica segundo Del Brutto et al. (2001) 11 .

As tomografias foram realizadas em todos os pacientes e a RM em cinco. As alterações observadas foram divididas em 6 tipos segundo a fase evolutiva da doença (Escobar, $1983^{13}$ e Machado et al., $1990^{14}$ com modificações): tipo 0 normal; tipo I- fase vesicular; tipo II- fase coloidal; tipo III- fase granular-nodular; tipo IV- fase granular-calcificada- e tipo V- presença de lesões sugestivas de 2 ou mais fases evolutivas. Quanto às alterações ventriculares, foram descritas independente dos grupos antes citados. Aqueles pacientes que apresentaram imagens tomográficas classificadas como tipo I, II, III ou V $(n=29)$ e alguns como tipo IV $(n=4)$, foram submetidos a estudo do LCR (aspecto, celularidade, dosagens de glicose e de proteínas e reações imunológicas para cisticercose). 0 exame do LCR foi considerado alterado quando apresentava mais que 5 células $/ \mathrm{mm}^{3}$, ou proteína elevada, ou glicose diminuída, ou pelo menos uma reação imunológica para cisticercose positiva (reação de fixação de complemento, imunofluorescência indireta, ou ELISA). Os pacientes que apresentaram convulsão foram submetidos a EEG. As convulsões foram classificadas segundo a classificação da International League Againsty Epilepsy (1981). O tratamento específico com praziquantel (PZQ), ou albendazol (ALB), associados ou não a dexametasona, foi instituído nos pacientes que apresentavam TC ou RM com lesões tipo I, II ou V e/ou LCR inflamatório. As doses utilizadas foram para o PZQ de $50 \mathrm{mg} / \mathrm{kg} / \mathrm{dia}$ e para o ALB de 15 a $20 \mathrm{mg} / \mathrm{kg} / \mathrm{dia}$, sendo o tempo de tratamento de 8 a 21 dias, conforme a droga utilizada. A dexametasona foi administrada na dose de 8 $\mathrm{mg}$ para adultos e $0,5 \mathrm{mg} / \mathrm{kg} / \mathrm{dia}$ para crianças, associada à droga cisticida por 8 dias. A TC de controle foi realizada nos pacientes com tomografias dos tipos I, II, III ou V , e por alguns do tipo IV. A RM de controle foi realizada por um paciente que apresentava imagens à TCC do tipo V.

Os dados coletados foram analisados após registro em planilha eletrônica e para as variáveis contínuas se calculou a média, mediana e o desvio padrão, e para as categóricas as medidas de frequência.

\section{RESULTADOS}

Dos 44 pacientes estudados, 23 (52,3\%) com neurocisticercose definitiva e $21(47,7 \%)$ com neurocisticercose provável, $31(70,5 \%)$ eram procedentes da zona urbana de Campina Grande e 13 (29,5\%) de outras cidades do Estado da Paraíba. Os demais dados demográficos estão descritos na Tabela 1. A idade de início dos sintomas variou de 8 meses a 58 anos, média de 20,6 $( \pm 14,3)$ anos, mediana de 17,5 anos; a maioria dos pacientes $(77,3 \%)$ tinha menos de 1 ano de doença.

Em 40 (90,9\%) pacientes a manifestação clínica inaugural da neurocisticercose foi a crise convulsiva, que ocorreu como único sintoma em $28(63,6 \%)$ e em $12(27,3 \%)$ associada a outros sintomas tais como: cefaléia em 10 (22,7\%), distúrbio psíquico em $3(6,8 \%)$, deficit motor em $3(6,8 \%)$, vômitos em 3 $(6,8 \%)$ e síndrome de hipertensão intracraniana (SHIC) em 2 (4,5\%). Como único sintoma, a cefaléia ocorreu em 4 pacientes $(9,1 \%)$. O tipo de convulsão mais frequente foi a generalizada, que ocorreu em 
Tabela 1. Dados demográficos dos pacientes portadores de neurocisticercose (Campina Grande-PB).

\begin{tabular}{|c|c|c|c|c|c|c|}
\hline \multirow[b]{3}{*}{ Características } & \multicolumn{4}{|c|}{ Gênero } & & \\
\hline & \multicolumn{2}{|c|}{ Masculino } & \multicolumn{2}{|c|}{ Feminino } & \multicolumn{2}{|c|}{ Total } \\
\hline & $\mathrm{n}$ & (\%) & $\mathrm{n}$ & (\%) & $\mathrm{n}$ & (\%) \\
\hline Idade (anos) & 24 & $(54,5)$ & 20 & $(45,5)$ & 44 & $(100,0)$ \\
\hline Média ( $\pm \mathrm{DP})$ & 22,7 & $( \pm 15,8)$ & 18,1 & $( \pm 12,1)$ & 20,6 & $( \pm 14,3)$ \\
\hline Mediana & 20 & & 15,5 & & 17,5 & \\
\hline \multicolumn{7}{|l|}{ Procedência } \\
\hline Campina Grande & 16,0 & $(36,4)$ & 15,0 & $(34,1)$ & 31,0 & $(70,5)$ \\
\hline Outras cidades $^{a}$ & 8,0 & $(18,2)$ & 5,0 & $(11,4)$ & 13,0 & $(29,5)$ \\
\hline \multicolumn{7}{|l|}{ Área de Residência } \\
\hline Urbana & 21,0 & $(47,7)$ & 17,0 & $(38,6)$ & 38,0 & $(86,2)$ \\
\hline Rural & 3,0 & $(6,8)$ & 3,0 & $(6,8)$ & 6,0 & $(13,6)$ \\
\hline \multicolumn{7}{|l|}{ Ocupação } \\
\hline Menor de 5 anos & 3,0 & $(6,8)$ & 3,0 & $(6,8)$ & 6,0 & $(13,6)$ \\
\hline Estudante & 7,0 & $(15,9)$ & 10,0 & $(22,7)$ & 17,0 & $(38,6)$ \\
\hline Outras profissões ${ }^{b}$ & 14,0 & $(31,8)$ & 7,0 & $(15,9)$ & 21,0 & $(47,7)$ \\
\hline \multicolumn{7}{|l|}{ Háb. Alimentar } \\
\hline Vegetais $^{1}$ & 18,0 & $(40,9)$ & 14,0 & $(31,8)$ & 32,0 & $(72,7)$ \\
\hline Carne de suino ${ }^{2}$ & 17,0 & $(38,6)$ & 13,0 & $(29,5)$ & 30,0 & $(68,2)$ \\
\hline
\end{tabular}

(a) Aroeiras, Água Branca, Boa Ventura, Cajazeiras, Marizópolis, Monteiro, Nova Floresta, Piancó, São João do Cariri, São Vicente do Seridó, Serra Branca, Sumé, Taperoá; (b) advogado, engenheiro, marchante, motorista, secretária, agente administrativo, técnico de laboratório, doméstica; (c) hábitos alimentares: (1) verduras e legumes; (2) carne de suíno e derivados.

$23(57,5 \%)$ dos casos, seguida da crise parcial com generalização secundária em 13 (32,5\%). O exame neurológico foi normal em $36(81,8 \%)$ e alterado em 7 (15,9\%). Uma criança de 7 anos e outra de 11 meses apresentaram a síndrome de hipertensão intracraniana clássica e foram os casos que mostraram maior gravidade. As formas clínicas encontradas entre os pacientes estudados foram: epiléptica em $28(63,6 \%)$, combinada em $10(22,7 \%)$, cefaléia em $4(9,1 \%)$ e meningoencefalítica em $2(4,6 \%)$.

Nas 44 TC realizadas ao diagnóstico, os achados mais frequentes foram: calcificações intraparenquimatosas em 26 (59,1\%), cistos viáveis em 16 (36,4\%), e cistos em degeneração em 14 (31,8\%) dos casos, que ocorreram de forma isolada ou associada. Na TC de controle (tratamento ou alta clínica) realizada em 23 pacientes, as lesões (do tipo I, II, III, ou V) evoluiram para lesões cicatriciais (calcificações ou alterações ventriculares) em 9 (39,1\%) pacientes, em 5 (21,7\%) permaneceram inalteradas, em um passou do tipo I para o tipo II e em $8(34,8 \%)$ normalizaram-se.

O estudo do LCR realizado em 29 pacientes, foi normal em $4(13,8 \%)$ e alterado em $25(86,2 \%)$. As alterações encontradas neste exame foram: pleocitose em $22(86,2 \%)$, que variou de 5 a 220 células, com predomínio linfomononuclear em 100\% dos casos e presença de eosinófilos em quatro $(18,2 \%)$. Hiperproteinorraquia foi observada em cinco $(20,8 \%)$, hipoglicorraquia em três $(12 \%)$ e positividade de reações imunológicas em 19 (64,3\%).

A terapêutica com drogas cisticidas PZQ ou ALB associadas ou não a dexametasona foi instituída em $23(52,3 \%)$ dos pacientes. O esquema terapêutico mais adotado foi a associação de ALB $(n=18)$ com dexametasona por período de 8 dias, com bons resultados. As 2 crianças que apresentaram SHIC necessitaram de uso mais prolongado e doses maiores do corticóide, e uma criança de 11 anos, mesmo após ser medicada com ALB por 3 vezes, com intervalo de 1 ano entre os esquemas, ainda persiste com cistos viáveis aos exames de neuroimagem, embora esteja assintomático.

Além do tratamento específico, os pacientes receberam tratamento sintomático e fisioterapia motora quando apresentaram deficit motor. Todos os pacientes evoluiram bem; apenas uma criança persiste com crises convulsivas de difícil controle.

\section{DISCUSSÃO}

Campina Grande é a segunda maior cidade do Estado da Paraíba (Nordeste brasileiro). Está localiza- 
da na região do Agreste paraibano, tem uma população de 360000 hab/IBGE 2000, sendo importante centro comercial e cultural do Estado. Possui 2 Universidades públicas e 2 particulares, rede hospitalar constituída por 17 clínicas e hospitais, a maioria deles conveniados do SUS (3.600 leitos). Possui 4 serviços de tomografia computadorizada e vários laboratórios de patologia clínica. O município é cortado por 3 rodovias que facilitam o acesso de grande número de pessoas provenientes de outras regiões do Estado, muitas delas com a assistência médica e hospitalar precárias que, diariamente, procuram a rede ambulatorial e ou hospitalar de Campina Grande.

Neste estudo, os 44 pacientes com diagnóstico de neurocisticercose, eram procedentes do Estado da Paraíba; a maioria deles $(70,5 \%)$ procedia da área urbana de Campina Grande e os demais de outras cidades do Estado, havendo neste grupo pacientes tanto da área urbana quanto da rural. Dos casos provenientes de outras cidades do Estado, alguns eram de cidades próximas, $<100 \mathrm{Km}$ de distância de Campina Grande; outros casos eram de cidades a mais de $100 \mathrm{Km}$, no sertão paraibano, evidenciando ampla distribuição desta doença no Estado da Paraíba. O predomínio da doença nas áreas urbanas reforça o que tem sido descrito em alguns trabalhos publicados nos últimos anos ${ }^{8,15}$ mostrando a inversão do que era descrito nos trabalhos mais antigos ${ }^{16}$. Este fato provavelmente se deve, pelo menos em parte, às migrações do homem do campo para cidades maiores, e às condições insatisfatórias em que grande parte da população vive, mesmo nas grandes metrópoles. A neurocisticercose, apesar de estar relacionada a condições sócio-econômicas insatisfatórias, foi, neste estudo, diagnosticada também em pacientes de nível sócio-econômico mais elevado, pois havia entre eles profissionais liberais e estudantes universitários. Isto sinaliza total descontrole do ciclo teníase/ cisticercose no Estado da Paraíba e condições sóciosanitárias inadequadas.

Não houve predomínio significativo entre os gêneros. $\mathrm{O}$ acometimento maior de pacientes jovens em plena fase produtiva, aumenta o risco de ficarem com limitações na sua vida profissional e social ${ }^{17}$. Estes dados estão de acordo com os descritos na literatura, que apresenta relatos de casos desde 1 ano até os 70 anos, sendo mais rara nos extre$\operatorname{mos}^{18}$ e predominando entre os 11 e $35 \operatorname{anos}^{19}$.

A elevada frequência da forma epiléptica apresentada no presente estudo $(63,6 \%)$ está de acordo com a maioria dos autores que a consideram a mais fre- quente, com taxas variando de $22 \%$ a $92 \%$, 4,8, , principalmente nas formas intraparenquimatosas, que constituiu a quase totalidade dos casos estudados. O predomínio das crises generalizadas difere do que é referido na maioria dos trabalhos publicados, os quais citam o predomínio das crises parciais com ou sem generalização secundária ${ }^{1,4,12,15,18,20}$ embora alguns poucos autores considerem como predominan-

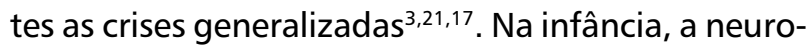
cisticercose pode ser responsável por até $91 \%$ dos casos de epilepsia ${ }^{20}$. Nesta casuística, a neurocisticercose foi causa de epilepsia em $73,7 \%$ dos menores de 15 anos. É uma cifra bastante elevada de acordo com o já descrito por outros autores. Isto leva a suspeitar do quanto estas crianças estão expostas aos agentes de endemicidade e vulneráveis a uma doença que pode também se apresentar com formas mais graves, (meningoencefalítica e HIC), como foi observado dentre os casos estudados, confirmando que também na infância e nas mulheres, a neurocisticercose poderá apresentar-se como formas graves e às vezes fatais ${ }^{4,6,16}$. Esta maior exposição das crianças aos fatores de endemicidade havia sido suspeitada por Pereira et al. ${ }^{10}$.

É sabido que o estudo do LCR e os exames de neuroimagem, são de grande importância não só para a confirmação diagnóstica desta doença como também para sua orientação terapêutica e acompanhamento evolutivo ${ }^{22,23}$, e que ambos se completam e um não exclui o outro ${ }^{24}$. Os achados tomográficos deste estudo são concordantes com os dados da literatura como descrito por Rodriguez-Carbajal \& Bolega-Duran apud Almeida, 1988, que afirmaram que $97 \%$ do diagnóstico de certeza pode ser obtido pela correlação clínico-radiológica. Embora o LCR seja importante para o diagnóstico, poderá numa primeira amostra ser normal em $20 \%$ dos casos $^{1}$, este fato pôde ser observado dentre os casos estudados, visto que, reações imunológicas foram reagentes em apenas $64,3 \%$ dos casos estudados. Isto pode ser explicado por provável baixa titulação de anticorpos, ou pela baixa sensibilidade e especificidade do método empregado, o que poderia ter sido resolvido com a realização de mais de uma reação. Desta forma, um resultado alterado confirma o diagnóstico, porém, um resultado negativo não o afasta'.

$\mathrm{O}$ achado de lesões em atividade e de lesões inativas na TC mostra que a neurocisticercose, tanto na forma ativa quanto na inativa, pode ser responsabilizada por epilepsia ativa em qualquer faixa etária25,26, já que os pacientes não apresentavam outras comorbidades que pudessem ser a causa de suas con- 
vulsões. O uso de drogas cisticidas no tratamento da neurocisticercose continua sendo tema controvertido. A maioria dos autores o indica nas formas parenquimatosas da doença, desde que existam lesões em atividade e a sintomatologia esteja presente ${ }^{23}$; outros, optam por deixar a doença evoluir espontaneamente (Jung, 1990 apud AndradeFilho $)^{17}$. Neste estudo, optou-se por tratar os pacientes que mostravam lesões em atividade e estavam sintomáticos; constatou-se que o tratamento foi eficaz na maioria dos pacientes e que as drogas utilizadas apresentam baixa toxicidade, como já havia sido relatado por outros autores ${ }^{28-30}$.

Em conclusão, os resultados obtidos neste estudo confirmam a ocorrência de neurocisticercose não só em Campina Grande mas também em várias regiões do Estado da Paraíba. A doença acomete indivíduos em qualquer faixa etária, sendo causa de epilepsia tanto na sua forma ativa como na inativa, preferencialmente em crianças e adultos jovens. $\mathrm{O}$ encontro desta afecção em diferentes ambientes sócio-econômicos, tanto na área urbana quanto na rural, evidencia o total descontrole do complexo teníase/cisticercose no Estado da Paraíba .

\section{REFERÊNCIAS}

1. Agapejev S. Neurocisticercose. In: Seminário Norte Rio Grandense sobre o Complexo Teníase/ Cisticercose. Natal, 2000;2-19.

2. Román G, SoteloJ,Del Brutto O, et al. A proposal to declare neurocysticercosis an international reportable disease. Bulletin of WHO, 2000;78:399-406.

3. White Junior AC. Neurocysticercosis: a major cause of neurological disease worldwide. Clin Infect Dis 1997;24:101-115.

4. Takayanagui OM. Neurocisticercose: I- Evolução clínica- laboratorial de 151 casos. Arq Neuropsiquiatr 1990:48:1-10.

5. Bruck I, Antoniuk AS, Wittig E, Accorsi A. Neurocisticercose na infância. Arq Neuropsiquiatr 1991;43-46.

6. Teive HAG, Minguetti G, Sasaki MGM, Lopes CE, Carvalho MTM, Szpeiter N. Neurocisticercose:artigo de revisão. Rev Brasil Neurol 1997;33:147-153.

7. Trelles JO \& Lazarte J. Cisticercosis Cerebral. Estudio clínico, histopatológico y parasitológico. Rev Neuropsiquiat (Lima) 1940;3:393-511.

8. Manreza MLG. Neurocisticercose na infância: Aspectos clínicos e do diagnóstico. Rev Hosp Clín Fac Med S.Paulo 1982;37:206-211.

9. Gonçalves-Coêlho TD, Coêlho MDG. Cerebral cysticercosis in Campina Grande, Paraíba-Northen Brazil: computerized tomography diagnosis importance. Arq Neuropsiquiatr 1996;54:94-97.
10. Pereira G, Assis MJM, Saksena KN. Enteroparasitoses em crianças de 0-8 anos de idade em favela de Campina Grande - Paraíba. In: Annais da $47^{a}$ Reunião Anual da SPBC, São Luis. 1995;Vol.II,108.

11. Del Brutto $\mathrm{OH}$, Rajshekhar V, White Jr AC et al. Proposed diagnostic criteria for neurocysticercosis. Neurology 2001;57:177-183.

12. Sotelo J, Guerreiro V, Rubio F. Neurocysticercosis: a new classification basead on active inactive forms. A study of 753 cases. Arch Intern Med1985;145:442-445.

13. Escobar A. The patology of neurocysticercosis.In Palacios EE, Rodrigues-Carbajal J, Taveras JM (eds). Cysticercosis of the central nervous system. Spring field: Charles Thomas, 1983

14. Machado LR, Nóbrega JPS, Barros NG, Livramento JA, Bacheschi LA, Spina-França A. Computed tomography in neurocysticercosis: a 10years long evolution analysis of 100 patients with na appraisal of a new classification. Arq Neuropsiquiatr1990;48;414-418.

15. Maretti MA. Neurocisticercose: estudo de 177 pacientes da região de São José do Rio Preto-SP. Dissertação de Mestrado. Faculdade de Medicina de São José do Rio Preto, São Paulo, 1999.

16. Takayanagui OM. Aspectos Clínicos da Neurocisticercose: análise de 500 casos. Dissertação de Mestrado. Faculdade de Medicina de Ribeirão Preto, São Paulo, 1980

17. Andrade-Filho AS. Neurocisticercose: aspectos clínicos, epidemiológicos e diagnóstico - estudo prospectivo de 157 pacientes na região Nordeste-Bahia. Universidade Federal Fluminense, Rio de Janeiro, 1997.

18. Wittig EO. Neurocisticercose: formas clínicas e aspectos anátomopatológicos. In Machado LR, Livramento JA, Spina-França A, Nóbrega JPS (eds). Neuroinfecção 96. São Paulo: Clínica Neurológica HC/ FMUSP, 1996;193-204.

19. Takayanagui OM, Leite JP. Neurocisticercose. Rev Soc Brasil Med Trop 2001;34:283-290.

20. Manreza MLG. Epilepsia e neurocisticercose. In Guerreiro CAM (ed) Epilepsia. 3Ed. São Paulo: Lemos 2000:255-264.

21. Del Brutto OH. Neurocisticercose. Neuropsiconews-Sociedade Brasileira de Informações de Patologias Médicas 2000;20:8-14.

22. Livramento JA. Contribuição de reações de imunofluorescência no líquido cefalorraqueano ao estudo da neurocisticercose. Arq Neuropsiquiatr 1981;39:261-277.

23. Nóbrega JPS. In Machado R, Livramento JA, Spina-França A, Nóbrega JPS (eds). Neuroinfeção 96. São Paulo : Clínica Neurológica HC/ FMUSP, 1996:219-226.

24. Spina-França A, Machado LR, Nóbrega JPS, et al. Praziquantel in the cerebrospinal fluid in neurocysticercosis. Arq Neuropsiquiatr 1985;43:444-448.

25. Comission on tropical diseases of the International League against Epilepsy. Relation between epilepsy and tropical diseases. Epilepsia 1994; 35:89-93.

26. Carpio A, Escobar A, Hauser WA. Cysticercosis and epilepsy:a critical review. Epilepsia 1998;39:1025-1040.

27. Marie SKN, Manreza MLG. Neurocisticercose: aspectos de neuroimagem. In Machado LR; Livramento JA, Spina-França A, Nóbrega JPS (eds). Neuroinfecção 96. São Paulo: Clínica Neurológica HC/ FMUSP, 1996: 213-218.

28. Takayanagui OM, Jardim E. Therapy for neurocysticercosis: comparasion between Albendazole e Praziquantel. Arch Neurol 1992;49:290-293.

29. Garcia HH,Gilman R, Horton J, et al. Albendazole therapy for neurocysticercosis:comparing 7 versus 14 days of treatment. Neurology 1997;48:1421-1427.

30. Robles C,Teutori NV, Sedano AM. Quimioterapia de la cisticercosis: resultados de 10 años ó más despues del seguimento. Gac Med Mex 1991;127-139. 